\title{
Stability studies of common biochemical analytes in serum separator tubes with or without gel barrier subjected to various storage conditions
}

\author{
Serap Cuhadar ${ }^{1 *}$, Ayşenur Atay ${ }^{1}$, Mehmet Koseoglu' ${ }^{1}$, Ahmet Dirican² ${ }^{2}$ Aysel Hur ${ }^{1}$ \\ ${ }^{1}$ Ataturk Training and Research Hospital, Department of Biochemistry and Clinical Biochemistry, Izmir, Turkey \\ ${ }^{2}$ Ataturk Training and Research Hospital, Department of Oncology, Izmir,Turkey \\ *Corresponding author: sdcuhadar@yahoo.com
}

\begin{abstract}
Introduction: The collected and shipped blood samples are exposed to a various extra-analytical factors prior to analysis. The aim of the study was to determine the stability of analytes in serum gel tubes and plain tubes exposed to a range of storage temperatures and times after centrifugation.

Materials and methods: Fifteen healthy volunteers were recruited and venous blood was collected into four tubes, two with and two without gel separator. Analyzing the baseline samples in $30 \mathrm{~min}$, all were stored at $4^{\circ} \mathrm{C}$ or $24^{\circ} \mathrm{C}$ for $6,12,18,24,30,36,48$ and 72 hours and 1 week. Sixteen biochemical anaytes were measured on each sample. Variations remained under the desirable bias considered as clinically insignificant.
\end{abstract}

Results: On day three, most analytes remained stable including albumin, protein, creatinine, cholesterol, triglycerides, gamma-glutamyl transferase (GGT), alkaline phosphatase (ALP), alanine aminotransferase (ALT), creatine kinase (CK), lactate dehydrogenase (LD) regardless of tube types. Glucose concentration decreased markedly $(P=0.001)$ beginning from the first hours of storage in plain serum. The stability maximized for the analytes including glucose, total bilirubin, urea nitrogen (BUN), uric acid stored at $4{ }^{\circ} \mathrm{C}$ in gel tubes. Aspartate aminotransferase (AST) activity increased significantly $(P=0.002)$ up to 48 -h, however bias was not significant clinically. High density lipoprotein $(H D L)$ concentration was stable in gel tubes at $24^{\circ} \mathrm{C}$, in plain tubes at $4^{\circ} \mathrm{C}$ stored up to 36 -h.

Conclusion: Serum gel or non-gel tubes might be used interchangeably for 11 analytes chilled or at $24^{\circ} \mathrm{C}$, whereas some restrictions must be applied for glucose, AST, BUN, HDL, and uric acid.

Keywords: blood specimen collection; enzyme stability; serum; storage; temperature.

\section{Introduction}

Collecting blood samples from individual laboratories and studying in one center has become more popular in recent years to ease health care costs. Accordingly, the extra-analytical factors affecting the analysis process such as different transportation conditions, prolonged storage at high or low temperature, improper handling (without frozen packs), are still sources of interest $(1,2)$. Though it is highly recommended to study tests from freshly drawn serum or plasma, delay in the testing process or reuse of the samples for missing results that may cause false concentrations are sometimes inevitable (3-5).
For many years the blood collection tubes have been used both with and without gel separator. During centrifugation, gel undergoes a temporary change in viscosity and lodges between the packed cells and the top serum layer. Advantages of separator tubes are rapid separation of serum from cellular constituents of blood, easy transportation, elimination the need of aliquoting serum, and reduction the aerosolization of hazardous substances (6).

However, blood collection tube additives also can be a source of analytical errors. Inorganic silicates, or ellagic acid, thrombin and tromboplastin are 
frequently used clot activators. Occasionally, clot activator particles may not pellet completely with the clot and may remain in the serum layer, causing interferences by coating pipetting devices especially during storage of the collection tube with improper storage temperatures $(7,8)$.

While interference mechanism of the tube components may be due to additives; where the interfering substance is present in the tube, chemical inhibition or activation in the assay by tube components, it is recommended to test tube component interference by collecting samples either at plain tubes and tubes with additives (9).

The information available for sample stability is incomplete and confusing, especially after prolonged contact of sera with clot or gel. Most of the studies were designed by assessing the stability of parameters prior to centrifugation or with whole blood stored for a few days. The main objective of our study was to evaluate whether the separator gel or clot in tubes influence the concentrations of 16 selected parameters in a clinically significant way. To mimic the delays in processing or shipping in the different tube types, we focused on four preanalytical conditions; a delay in processing of plain serum at $4{ }^{\circ} \mathrm{C}$, and at $24^{\circ} \mathrm{C}$, and a delay in processing of serum in gel tube at $4^{\circ} \mathrm{C}$, and at 24 ${ }^{\circ} \mathrm{C}$ up to one week of storage in ten time intervals. This study may help to verify the optimum stability conditions for mailed specimens before reaching the laboratory.

\section{Materials and methods}

\section{Subjects}

This experimental study was carried out in March 2010. The study group consisted of fifteen voluntary physicians and laboratory technicians (9 females and 6 males, age range 24-45 years), who gave written informed consent form for testing. The procedures we used were in accordance with the guidelines of the Helsinki Declaration on human experimentation.

\section{Blood sampling}

Blood was collected by using Vacuette ${ }^{\odot}$ Standard tube holder and Vacuette 21 -gauge, $0.80 \times 38 \mathrm{~mm}$ multisample needle (Vacuette, Greiner Bio-One, Kremsmünster, Austria).
Blood specimens were drawn into following types of plastic tubes: Vacuette ${ }^{\oplus}$ Tube Serum No Gel 9 $\mathrm{mL}$ (Ref. No. 455092) (Serum Plain) and Vacuette Tube Serum Gel Separator 8 mL (Ref. No. 455071) (Serum Gel).

Fasting venous blood was randomly collected in the morning from each participant by a single venipuncture to control for any draw order bias and the tubes were filled completely. Blood (totally, 34 $\mathrm{mL}$ ) was collected from each volunteer using two serum separator tubes with gel and two without gel. All were categorized according to tube types and storage temperatures:

- Group I ( $N=15$ ) Serum Gel at $24^{\circ} \mathrm{C}$ (room temperature defined as RT);

- Group II $(\mathrm{N}=15)$ Serum Gel at $4{ }^{\circ} \mathrm{C}$ (chilled);

- Group III (N=15) Serum Plain at $24^{\circ} \mathrm{C}$;

- Group IV $(\mathrm{N}=15)$ Serum Plain at $4^{\circ} \mathrm{C}$.

Sera were allowed to clot for $30 \mathrm{~min}$ at RT and then centrifuged at $2200 \times \mathrm{g}$ for $10 \mathrm{~min}$. Immediately following centrifugation, all specimens were analyzed within 30-min to obtain the "0 hours $=\mathrm{T}_{0}$ " (baseline) values. Tubes of each group were then stored at particular storage temperatures (RT or chilled) and analyzed at different time intervals (6-, 12-, 18-, 24-, 30-, 36-, 48-, 72-hour and 1 week). To evaluate the stability of analytes in serum separator tubes, sera were stored on the gel or on the clot during the process. The tubes were kept avoiding from light exposure at RT or chilled during all storage times up to one week. To avoid evaporation, sample tubes were kept closed until analysis.

\section{Methods}

The following analytes were studied:

- metabolites: glucose, total bilirubin, creatinine, BUN, uric acid;

- proteins: total protein, albumin;

- lipids: triglycerides, cholesterol, HDL;

- enzymes: ALP, ALT, AST, CK, GGT, and LD.

All assays were performed on the Abbott Aeroset (Abbott, Wiesbaden, Germany), according to the manufacturer's specifications by using proprietary reagents at the Department of Biochemistry Laboratory of Ataturk Training and Research Hospital in Izmir, Turkey.

Intra-assay analytical CV's were determined by two levels of control materials (Bio-Rad Laboratories, 
Milano, Italy) ( $\mathrm{N}=10$, from each level on the same plate) on the same day before biochemical analysis (Table 1). Additionally, for accuracy and internal quality check, two levels of control materials and the test samples were assayed in the same analytical run at each assay point during the process. All values of QC samples for analytes were within \pm 2 SD (standard deviation) of their respective target means during the entire investigation.

\section{Statistical analysis}

In the current study, to determine time dependent changes, the median from all 15 volunteers for each analyte was obtained for each time interval at each group. Statistically significant changes were determined by non-parametric Friedman test for repeated-measures to examine the influence of storage time. Finding the significant differences $(P<0.05)$, Wilcoxon signed-rank test was used for those groups. Post-hoc analysis was conducted with a Bonferroni correction applied, resulting in a significance level set at $P<0.006(0.05 / 9$ $=0.0055$ ). All statistical analyses were performed using SPSS for Windows, version 15.0 (SPSS Inc., Chicago, IL, USA). To determine the clinically signif- icant variations the percentage relative bias from the baseline sample was calculated by the formula:

$$
[(\mathrm{CX}-\mathrm{C} 1) / \mathrm{C} 1)] \times 100 .
$$

$\mathrm{C} 1$ : the median result of the $\mathrm{T}_{0}$ sample $(\mathrm{N}=15)$, $\mathrm{Cx}$ : the median result of the stored sample $(\mathrm{N}=$ 15).

Percentage relative bias for paired groups $\left(T_{0}-T_{6}\right.$ $T_{0}-T_{12}, T_{0}-T_{18}$, etc.) was then compared with the current analytical quality specifications for desirable bias taken from the Westgard QC (10), which was first published at 1999 and updated at 2012 by Ricos et al.

\section{Results}

The results of the present investigation at $T_{0}$ are presented in Table 2 along with the reference intervals for each analyte.

The statistical analysis of serum gel / clot contact time and temperature effects is presented in Table 3. The tests that were suitable for analysis on the third day of storage in all groups were cholesterol, triglycerides, creatinine, CK, ALP, ALT, GGT LD

TABLE 1. Intra-assay coefficient of variations determined at two levels (Level 1 and 2).

\begin{tabular}{lcccc}
\hline Analyte & CV 1 (\%) & Level 1 & CV 2 (\%) & Level 2 \\
\hline Alanine aminotransferase (U/L) & 1.5 & 37 & 0.7 & 110 \\
Albumin (g/L) & 1.8 & 39.8 & 2.0 & 23.7 \\
Alkaline phosphatase (U/L) & 2.1 & 93 & 0.4 & 415 \\
Aspartate aminotransferase (U/L) & 2.1 & 37 & 0.5 & 186 \\
Bilirubin (total) ( $\mu$ mol/L) & 1.5 & 17.7 & 0.5 & 90 \\
Cholesterol (mmol/L) & 0.6 & 6.84 & 0.6 & 2.59 \\
Creatinine ( $\mu$ mol/L) & 2.2 & 181 & 0.7 & 539 \\
Creatine kinase (U/L) & 0.9 & 135 & 0.3 & 431 \\
Glucose (mmol/L) & 0.4 & 5 & 0.7 & 16.5 \\
Gamma -glutamyl transferase (U/L) & 0.7 & 61.2 & 0.5 & 156 \\
HDL-cholesterol (mmol/L) & 3.3 & 1.89 & 5.3 & 0.81 \\
Lactate dehydrogenase (U/L) & 1.0 & 202 & 0.8 & 372 \\
Protein (total) $(\mathrm{g} / \mathrm{L})$ & 0.6 & 67.8 & 1.3 & 43.3 \\
Triglyceride (mmol/L) & 0.4 & 2.07 & 1.0 & 0.95 \\
Urea nitrogen (mmol/L) & 0.0 & 5.36 & 1.6 & 16.83 \\
Uric acid ( $\mu$ mol/L) & 0.8 & 302 & 0.8 & 592 \\
\hline For CV measurement, 10 samples were assayed on each level on the same plate. & &
\end{tabular}


TABLE 2. Methods, $T_{0}$ (median and interquartile range) values and reference intervals.

\begin{tabular}{|c|c|c|c|}
\hline Analyte & Method & Median (IQR) & Reference interval* \\
\hline Alanine aminotransferase (U/L) & UV without P5P & $15(9-65)$ & $3-44$ \\
\hline Albumin (g/L) & Bromcresol green & $44(40-48)$ & $35-52^{\S}$ \\
\hline Alkaline phosphatase (U/L) & PNPP & $69(37-135)$ & $44-134$ \\
\hline Aspartate aminotransferase (U/L) & UV without P5P & $14(8-31)$ & $10-32$ \\
\hline Bilirubin (total) ( $\mu \mathrm{mol} / \mathrm{L})$ & Diazonium ion & $10.9(6.3-56.8)$ & $5-21^{\S}$ \\
\hline Cholesterol (mmol/L) & CHOD-PAP & $4.44(3.52-6.92)$ & $3.13-6.97$ \\
\hline Creatinine $(\mu \mathrm{mol} / \mathrm{L})$ & Alkaline picrate & $71(44-97)$ & $56-101$ \\
\hline Creatine kinase (U/L) & UV-NAC activated & $84(27-170)$ & $\leq 145^{\S}$ \\
\hline Glucose (mmol/L) & Hexokinase & $5.25(4.05-7.33)$ & $4.00-5.83$ \\
\hline Gamma -glutamyl transferase (U/L) & $\gamma$-glutamyl-carboxy nitroanilide & $20(10-49)$ & $<38^{\S}$ \\
\hline HDL-cholesterol (mmol/L) & Direct, non-immunologic & $1.41(0.67-2.10)$ & $0.8-2.0$ \\
\hline Lactate dehydrogenase (U/L) & Lactate-pyruvate, UV & $164(123-222)$ & $<247^{\S}$ \\
\hline Protein (total) (g/L) & Biuret & $71(67-80)$ & $66-83^{\S}$ \\
\hline Triglyceride (mmol/L) & GPO-PAP & $0.84(0.54-3.01)$ & $0.4-3.5$ \\
\hline Urea nitrogen (mmol/L) & Urease, UV & $4.5(2.9-7.1)$ & $2.1-6.8$ \\
\hline Uric acid ( $\mu \mathrm{mol} / \mathrm{L})$ & Uricase, PAP & $232(155-411)$ & $130-480$ \\
\hline
\end{tabular}

* method-specific reference intervals established in-house from analysis of healthy adults (23);

$\S$ reference intervals established by manufacturer (Abbott Diagnostics).

TABLE 3. Comparison of tubes with gel and without gel for analytes at room temperature or 4 degrees centigrade according to time of analysis (hour, week).

\begin{tabular}{|c|c|c|c|c|c|c|c|c|c|c|c|c|c|}
\hline 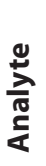 & 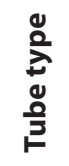 & 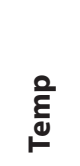 & 气n & $\frac{5}{0}$ & $\stackrel{\mathbf{N}}{\mathfrak{I}}$ & $\underset{\infty}{\infty}$ & $\stackrel{\mathbf{d}}{\mathbf{N}}$ & ¿) & $\frac{c}{0}$ & $\underset{+\infty}{\infty}$ & $\hat{\mathbb{N}}$ & 3 & 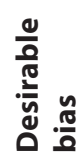 \\
\hline \multirow{12}{*}{ 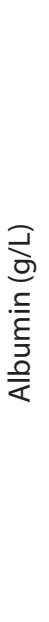 } & & & & 45 & 44 & 44 & 44 & 43 & 44 & 44 & 44 & 44 & \multirow{12}{*}{$1.3 \%$} \\
\hline & & $24^{\circ} \mathrm{C}$ & 44 & $(2.3 \%)$ & $(0.0 \%)$ & $(0.0 \%)$ & $(0.0 \%)$ & $(-2.3 \%)$ & $(0.0 \%)$ & $(0.0 \%)$ & $(0.0 \%)$ & $(0.0 \%)$ & \\
\hline & & & & $P=0.806$ & $P=0.138$ & $\mathrm{P}=0.372$ & $P=0.495$ & $P=0.083$ & $P=0.145$ & $P=0.224$ & $P=0.233$ & $P=0.124$ & \\
\hline & gei & & & 44 & 44 & 44 & 44 & 43 & 43 & 44 & 44 & 44 & \\
\hline & & $4^{\circ} \mathrm{C}$ & 44 & $(0.0 \%)$ & $(0.0 \%)$ & $(0.0 \%)$ & $(0.0 \%)$ & $(-2.3 \%)$ & $(-2.3 \%)$ & $(0.0 \%)$ & $(0.0 \%)$ & $(0.0 \%)$ & \\
\hline & & & & $P=0.005$ & $P=0.053$ & $P=0.058$ & $P=1.000$ & $P=0.027$ & $P=0.162$ & $P=0.083$ & $P=0.076$ & $P=0.052$ & \\
\hline & & & & 43 & 45 & 44 & 44 & 45 & 44 & 44 & 44 & 44 & \\
\hline & \multirow{5}{*}{$\begin{array}{l}\text { w/o } \\
\text { gel }^{*}\end{array}$} & $24^{\circ} \mathrm{C}$ & 44 & $(-2.3 \%)$ & $(2.3 \%)$ & $(0.0 \%)$ & $(0.0 \%)$ & (2.3\%) & $(0.0 \%)$ & $(0.0 \%)$ & $(0.0 \%)$ & $(0.0 \%)$ & \\
\hline & & & & $P=0.593$ & $P=0.249$ & $\mathrm{P}=0.279$ & $P=0.589$ & $P=0.100$ & $P=0.598$ & $P=0.519$ & $P=0.219$ & $P=0.250$ & \\
\hline & & & & 44 & 45 & 44 & 45 & 44 & 44 & 44 & 44 & 45 & \\
\hline & & $4^{\circ} \mathrm{C}$ & 44 & $(0.0 \%)$ & $(2.3 \%)$ & $(0.0 \%)$ & (2.3\%) & $(0.0 \%)$ & $(0.0 \%)$ & $(0.0 \%)$ & $(0.0 \%)$ & (2.3\%) & \\
\hline & & & & $P=1.000$ & $P=0.406$ & $P=0.124$ & $P=0.578$ & $P=0.490$ & $P=0.030$ & $P=0.113$ & $P=0.084$ & $P=0.406$ & \\
\hline
\end{tabular}




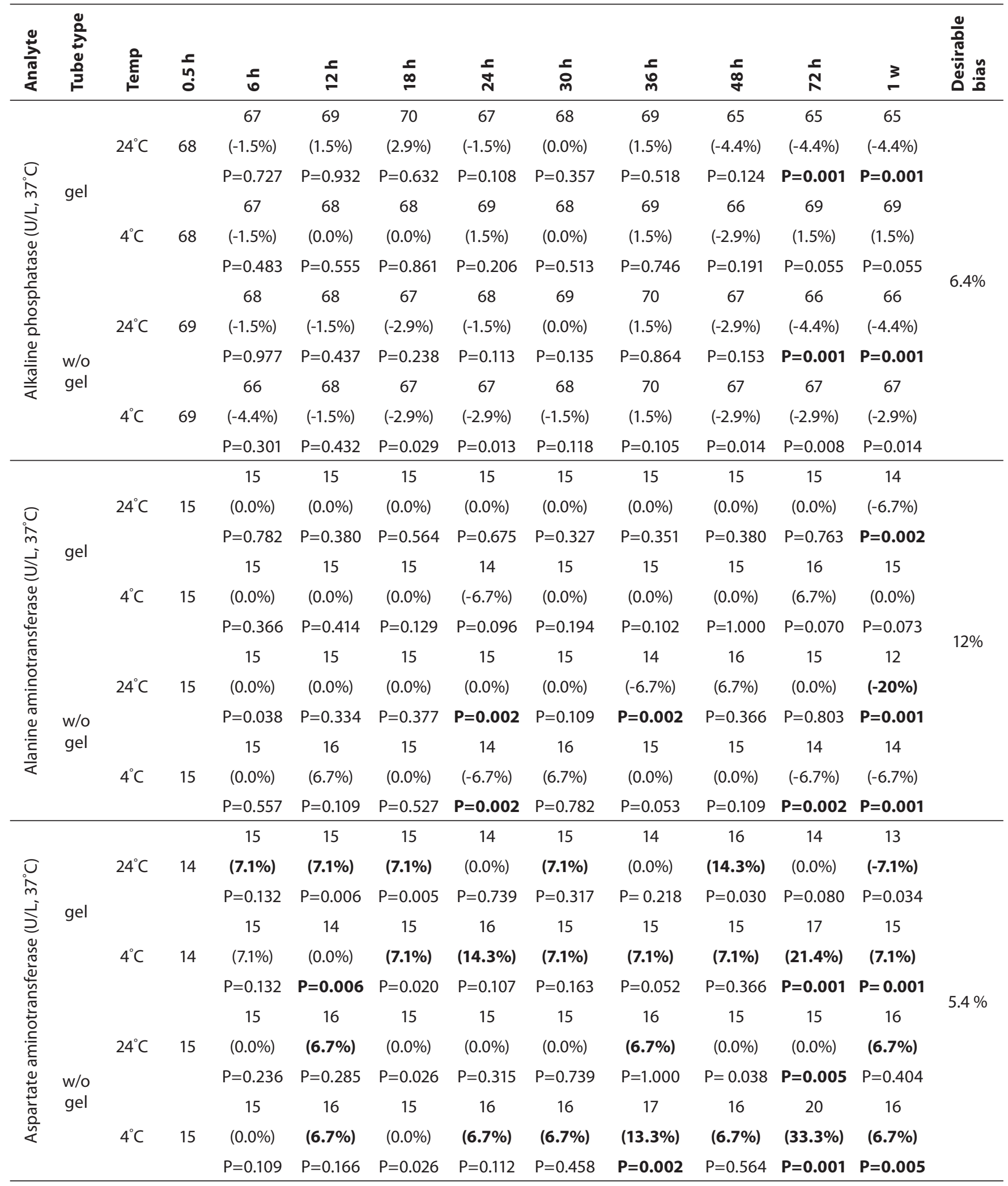




\begin{tabular}{|c|c|c|c|c|c|c|c|c|c|c|c|c|c|}
\hline 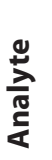 & 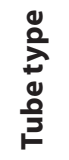 & $\frac{\stackrel{\varrho}{E}}{\stackrel{E}{\varrho}}$ & $\begin{array}{l}\frac{c}{n} \\
0\end{array}$ & $\frac{1}{6}$ & $\stackrel{\mathbf{N}}{\mathbf{N}}$ & $\frac{c}{\infty}$ & $\frac{c}{d}$ & 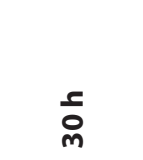 & $\frac{c}{b}$ & $\underset{+}{\infty}$ & $\stackrel{\mathbf{N}}{\mathbf{N}}$ & 3 & 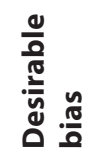 \\
\hline \multirow{12}{*}{ 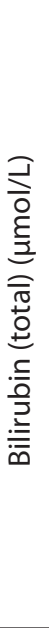 } & \multirow{6}{*}{ gel } & & & 10.8 & 10.8 & 9.9 & 10.6 & 10.3 & 10.6 & 10.9 & 11.1 & 11.6 & \multirow{12}{*}{$11.4 \%$} \\
\hline & & $24^{\circ} \mathrm{C}$ & 10.9 & $(-1.6 \%)$ & $(-1.6 \%)$ & $(-9.3 \%)$ & $(-3.1 \%)$ & $(-6.2 \%)$ & $(-3.1 \%)$ & (0.0\%) & (1.7\%) & (6.3\%) & \\
\hline & & & & $P=0.001$ & $P=0.001$ & $P=0.001$ & $P=0.001$ & $P=0.001$ & $P=0.001$ & $P=0.007$ & $P=0.136$ & $P=0.589$ & \\
\hline & & & & 10.4 & 10.3 & 10.3 & 10.4 & 10.3 & 10.3 & 10.3 & 10.3 & 11.1 & \\
\hline & & $4^{\circ} \mathrm{C}$ & 10.9 & $(-4.7 \%)$ & $(-6.2 \%)$ & $(-6.2 \%)$ & $(-4.7 \%)$ & $(-6.2 \%)$ & $(-6.2 \%)$ & $(-6.2 \%)$ & $(-6.2 \%)$ & (1.7\%) & \\
\hline & & & & $P=0.001$ & $P=0.001$ & $P=0.001$ & $P=0.001$ & $P=0.001$ & $P=0.001$ & $P=0.001$ & $P=0.002$ & $P=0.006$ & \\
\hline & \multirow{6}{*}{$\begin{array}{l}\text { w/o } \\
\text { gel }\end{array}$} & & & 10.3 & 10.3 & 9.6 & 10.3 & 10.3 & 9.9 & 9.9 & 9.9 & 9.9 & \\
\hline & & $24^{\circ} \mathrm{C}$ & 10.9 & $(-6.2 \%)$ & $(-6.2 \%)$ & $(-12.4 \%)$ & $(-6.2 \%)$ & $(-6.2 \%)$ & $(-9.3 \%)$ & $(-9.3 \%)$ & $(-9.3 \%)$ & $(-9.3 \%)$ & \\
\hline & & & & $P=0.005$ & $P=0.001$ & $P=0.001$ & $P=0.003$ & $P=0.001$ & $P=0.001$ & $P=0.009$ & $P=0.030$ & $P=0.010$ & \\
\hline & & & & 10.6 & 9.9 & 9.1 & 10.8 & 10.3 & 10.6 & 10.6 & 9.9 & 12.3 & \\
\hline & & $4^{\circ} \mathrm{C}$ & 10.9 & $(-3.1 \%)$ & $(-9.3 \%)$ & $(-17.2 \%)$ & $(-1.6 \%)$ & $(-6.2 \%)$ & $(-3.1 \%)$ & $(-3.1 \%)$ & $(-9.3 \%)$ & (12.5\%) & \\
\hline & & & & $P=0.007$ & $P=0.001$ & $P=0.001$ & $P=0.200$ & $P=0.001$ & $P=0.972$ & $P=0.141$ & $P=0.019$ & $P=0.001$ & \\
\hline \multirow{12}{*}{ 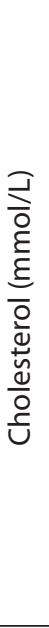 } & \multirow{6}{*}{ gel } & & & 4.46 & 4.43 & 4.48 & 4.58 & 4.56 & 4.46 & 4.53 & 4.48 & 4.58 & \multirow{12}{*}{$4 \%$} \\
\hline & & $24^{\circ} \mathrm{C}$ & 4.43 & $(0.6 \%)$ & $(0.0 \%)$ & (1.2\%) & (3.5\%) & $(2.9 \%)$ & $(0.6 \%)$ & (2.4\%) & (1.2\%) & (3.5\%) & \\
\hline & & & & $P=0.001$ & $P=0.014$ & $P=0.021$ & $P=0.030$ & $P=0.004$ & $P=0.001$ & $P=0.002$ & $P=0.001$ & $P=0.062$ & \\
\hline & & & & 4.50 & 4.50 & 4.50 & 4.50 & 4.50 & 4.50 & 4.50 & 4.50 & 4.50 & \\
\hline & & $4^{\circ} \mathrm{C}$ & 4.40 & (1.8\%) & (1.8\%) & $(0.7 \%)$ & (1.8\%) & (2.3\%) & $(2.3 \%)$ & (1.1\%) & (1.8\%) & (1.8\%) & \\
\hline & & & & $P=0.016$ & $\mathrm{P}=0.011$ & $P=0.001$ & $P=0.002$ & $P=0.068$ & $P=0.068$ & $P=0.047$ & $P=0.001$ & $P=0.019$ & \\
\hline & \multirow{6}{*}{$\begin{array}{l}\text { w/o } \\
\text { gel }\end{array}$} & & & 4.43 & 4.48 & 4.58 & 4.58 & 4.46 & 4.51 & 4.53 & 4.56 & 4.61 & \\
\hline & & $24^{\circ} \mathrm{C}$ & 4.46 & $(-0.7 \%)$ & (0.5\%) & $(2.7 \%)$ & (2.7\%) & $(0.0 \%)$ & (1.1\%) & (1.6\%) & $(2.2 \%)$ & (3.4\%) & \\
\hline & & & & $P=0.771$ & $P=0.012$ & $P=0.001$ & $P=0.001$ & $P=0.002$ & $P=0.005$ & $P=0.001$ & $P=0.001$ & $P=0.001$ & \\
\hline & & & & 4.50 & 4.50 & 4.40 & 4.50 & 4.50 & 4.40 & 4.50 & 4.50 & 4.60 & \\
\hline & & $4^{\circ} \mathrm{C}$ & 4.50 & (1.6\%) & (1.1\%) & $(-0.7 \%)$ & (0.0\%) & $(0.5 \%)$ & $(-0.7 \%)$ & $(1.1 \%)$ & $(0.0 \%)$ & (2.7\%) & \\
\hline & & & & $P=1.000$ & $P=0.111$ & $P=0.406$ & $P=0.580$ & $P=0.020$ & $P=0.032$ & $P=0.002$ & $P=0.001$ & $P=0.001$ & \\
\hline \multirow{12}{*}{ 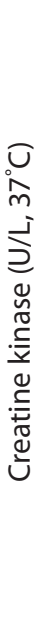 } & \multirow{6}{*}{ gel } & & & 83 & 84 & 83 & 83 & 83 & 82 & 82 & 92 & 97 & \multirow{12}{*}{$11.5 \%$} \\
\hline & & $24^{\circ} \mathrm{C}$ & 83 & (0.0\%) & $(1.2 \%)$ & $(0.0 \%)$ & (0.0\%) & $(0.0 \%)$ & $(-1.2 \%)$ & $(-1.2 \%)$ & (10.8\%) & (16.9\%) & \\
\hline & & & & $P=0.593$ & $P=0.548$ & $\mathrm{P}=0.282$ & $P=0.249$ & $P=0.471$ & $P=0.472$ & $P=0.871$ & $P=0.186$ & $P=0.045$ & \\
\hline & & & & 82 & 83 & 84 & 84 & 83 & 81 & 82 & 83 & 81 & \\
\hline & & $4^{\circ} \mathrm{C}$ & 83 & $(-1.2 \%)$ & (0.0\%) & $(1.2 \%)$ & (1.2\%) & $(0.0 \%)$ & $(-2.4 \%)$ & $(-1.2 \%)$ & $(0.0 \%)$ & $(-2.4 \%)$ & \\
\hline & & & & $P=0.346$ & $P=0.544$ & $P=0.318$ & $P=0.110$ & $P=0.840$ & $P=0.747$ & $P=0.609$ & $P=0.142$ & $P=0.401$ & \\
\hline & \multirow{6}{*}{$\begin{array}{l}\text { w/o } \\
\text { gel }\end{array}$} & & & 83 & 85 & 86 & 85 & 84 & 86 & 85 & 86 & 105 & \\
\hline & & $24^{\circ} \mathrm{C}$ & 84 & $(-1.2 \%)$ & (1.2\%) & $(2.4 \%)$ & $(1.2 \%)$ & $(0.0 \%)$ & $(2.4 \%)$ & $(1.2 \%)$ & (2.4\%) & (25\%) & \\
\hline & & & & $P=0.008$ & $P=0.714$ & $\mathrm{P}=0.716$ & $\mathrm{P}=0.078$ & $P=0.593$ & $P=0.297$ & $P=0.593$ & $\mathrm{P}=0.233$ & $P=0.001$ & \\
\hline & & & & 82 & 83 & 84 & 84 & 83 & 81 & 82 & 83 & 81 & \\
\hline & & $4^{\circ} \mathrm{C}$ & 83 & $(-1.2 \%)$ & $(0.0 \%)$ & $(1.2 \%)$ & $(1.2 \%)$ & $(0.0 \%)$ & $(-2.4 \%)$ & $(-1.2 \%)$ & (0.0\%) & $(-2.4 \%)$ & \\
\hline & & & & $P=0.175$ & $P=0.816$ & $P=0.399$ & $P=0.336$ & $P=1.000$ & $P=0.305$ & $P=0.088$ & $P=0.006$ & $P=0.003$ & \\
\hline
\end{tabular}




\begin{tabular}{|c|c|c|c|c|c|c|c|c|c|c|c|c|c|}
\hline 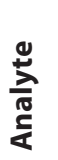 & 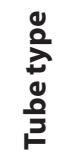 & $\stackrel{\text { 을 }}{\stackrel{E}{\sigma}}$ & 而 & $\frac{1}{6}$ & $\stackrel{\Sigma}{N}$ & $\stackrel{\infty}{\infty}$ & $\frac{8}{d}$ & হ & $\frac{c}{0}$ & $\underset{⿱ 亠}{\infty}$ & $\stackrel{\Sigma}{N}$ & 3 & 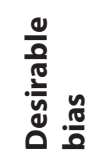 \\
\hline \multirow{12}{*}{ 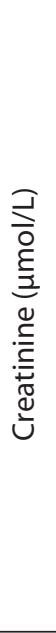 } & \multirow{6}{*}{ gel } & \multirow{3}{*}{$24^{\circ} \mathrm{C}$} & \multirow{3}{*}{70.7} & 70.7 & 70.7 & 70.7 & 61.9 & 70.7 & 70.7 & 70.7 & 70.7 & 70.7 & \multirow{12}{*}{$4.0 \%$} \\
\hline & & & & $(0.0 \%)$ & (0.0\%) & $(0.0 \%)$ & $(-12.5 \%)$ & (0.0\%) & (0.0\%) & (0.0\%) & (0.0\%) & $(0.0 \%)$ & \\
\hline & & & & $P=0.317$ & $P=0.705$ & $P=0.102$ & $P=0.705$ & $P=0.655$ & $P=0.655$ & $P=1.000$ & $P=0.655$ & $P=0.102$ & \\
\hline & & \multirow{6}{*}{$24^{\circ} \mathrm{C}$} & \multirow{3}{*}{70.7} & 70.7 & 70.7 & 70.7 & 70.7 & 70.7 & 70.7 & 70.7 & 70.7 & 70.7 & \\
\hline & & & & (0.0\%) & (0.0\%) & (0.0\%) & $(0.0 \%)$ & (0.0\%) & (0.0\%) & $(0.0 \%)$ & (0.0\%) & (0.0\%) & \\
\hline & & & & $P=1.000$ & $\mathrm{P}=0.705$ & $P=1.000$ & $\mathrm{P}=0.655$ & $P=0.705$ & $P=0.180$ & $P=1.000$ & $P=1.000$ & $P=0.655$ & \\
\hline & \multirow{6}{*}{$\begin{array}{l}\text { w/o } \\
\text { gel }\end{array}$} & & \multirow{6}{*}{70.7} & 70.7 & 70.7 & 70.7 & 70.7 & 70.7 & 70.7 & 70.7 & 70.7 & 70.7 & \\
\hline & & & & (0.0\%) & (0.0\%) & (0.0\%) & (0.0\%) & (0.0\%) & (0.0\%) & (0.0\%) & (0.0\%) & (0.0\%) & \\
\hline & & & & $\mathrm{P}=0.317$ & $P=0.157$ & $\mathrm{P}=0.020$ & $\mathrm{P}=0.059$ & $P=0.096$ & $P=0.180$ & $P=0.005$ & $P=1.000$ & $P=0.180$ & \\
\hline & & \multirow{3}{*}{$4^{\circ} \mathrm{C}$} & & 70.7 & 70.7 & 70.7 & 70.7 & 70.7 & 70.7 & 70.7 & 61.9 & 70.7 & \\
\hline & & & & (0.0\%) & (0.0\%) & (0.0\%) & (0.0\%) & (0.0\%) & (0.0\%) & $(0.0 \%)$ & $(-12.5 \%)$ & (0.0\%) & \\
\hline & & & & $P=0.480$ & $P=0.257$ & $P=0.011$ & $P=0.180$ & $P=0.180$ & $\mathrm{P}=0.020$ & $P=0.317$ & $P=0.002$ & $P=0.102$ & \\
\hline \multirow{12}{*}{ 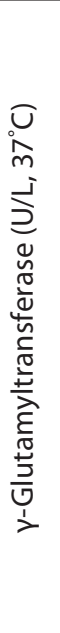 } & \multirow{6}{*}{ gel } & \multirow{3}{*}{$24^{\circ} \mathrm{C}$} & \multirow{3}{*}{20} & 19 & 20 & 19 & 20 & 20 & 19 & 19 & 19 & 21 & \multirow{12}{*}{$10.8 \%$} \\
\hline & & & & $(-5 \%)$ & $(0.0 \%)$ & $(-5 \%)$ & $(0.0 \%)$ & (0.0\%) & $(-5 \%)$ & $(-5 \%)$ & $(-5 \%)$ & (5\%) & \\
\hline & & & & $P=0.206$ & $P=0.206$ & $P=0.058$ & $P=0.627$ & $P=0.190$ & $\mathrm{P}=0.903$ & $\mathrm{P}=0.386$ & $\mathrm{P}=0.429$ & $P=0.206$ & \\
\hline & & \multirow{7}{*}{$24^{\circ} \mathrm{C}$} & \multirow{3}{*}{20} & 19 & 18 & 19 & 19 & 20 & 19 & 20 & 18 & 18 & \\
\hline & & & & $(-5 \%)$ & $(-10 \%)$ & $(-5 \%)$ & $(-5 \%)$ & (0.0\%) & $(-5 \%)$ & $(0.0 \%)$ & $(-10 \%)$ & $(-10 \%)$ & \\
\hline & & & & $P=0.130$ & $P=0.013$ & $P=0.366$ & $P=0.739$ & $P=0.206$ & $P=0.791$ & $P=0.564$ & $P=0.021$ & $P=0.290$ & \\
\hline & \multirow{6}{*}{$\begin{array}{l}\text { w/o } \\
\text { gel }\end{array}$} & & & 20 & 20 & 19 & 19 & 19 & 20 & 19 & 20 & 21 & \\
\hline & & & 19 & (5.3\%) & (5.3\%) & $(0.0 \%)$ & (0.0\%) & $(0.0 \%)$ & (5.3\%) & (0.0\%) & (5.3\%) & (10.5\%) & \\
\hline & & & & $P=0.366$ & $P=0.166$ & $P=0.763$ & $P=0.102$ & $P=0.160$ & $P=0.001$ & $P=0.627$ & $P=0.107$ & $P=0.002$ & \\
\hline & & & & 19 & 18 & 19 & 20 & 20 & 21 & 19 & 20 & 20 & \\
\hline & & $4^{\circ} \mathrm{C}$ & 19 & (0.0\%) & $(-5.3 \%)$ & (0.0\%) & (5.3\%) & (5.3\%) & (10.5\%) & (0.0\%) & (5.3\%) & (5.3\%) & \\
\hline & & & & $P=0.782$ & $P=0.730$ & $P=0.414$ & $P=0.159$ & $P=0.773$ & $P=0.002$ & $P=0.271$ & $P=0.272$ & $P=0.002$ & \\
\hline \multirow{12}{*}{ 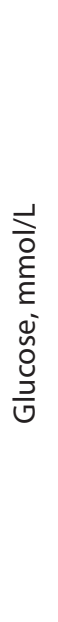 } & \multirow{6}{*}{ gel } & & & 5.22 & 5.27 & 5.16 & 5.00 & 5.22 & 4.94 & 4.94 & 4.88 & 4.77 & \multirow{12}{*}{$2.2 \%$} \\
\hline & & $24^{\circ} \mathrm{C}$ & 5.27 & $(-1.0 \%)$ & (0.0\%) & $(-2.1 \%)$ & $(-5.3 \%)$ & $(-1.0 \%)$ & $(-6.3 \%)$ & $(-6.3 \%)$ & (-7.4\%) & $(-9.5 \%)$ & \\
\hline & & & & $P=0.026$ & $P=0.334$ & $P=0.400$ & $P=0.003$ & $P=0.003$ & $P=0.003$ & $P=0.001$ & $P=0.001$ & $P=0.001$ & \\
\hline & & & & 5.27 & 5.33 & 5.27 & 5.22 & 5.22 & 5.16 & 5.11 & 5.05 & 5.00 & \\
\hline & & $4^{\circ} \mathrm{C}$ & 5.27 & $(0.0 \%)$ & (1.1\%) & $(0.0 \%)$ & $(-1.1 \%)$ & $(-1.1 \%)$ & $(-2.1 \%)$ & $(-3.0 \%)$ & $(-4.2 \%)$ & $(-5.1 \%)$ & \\
\hline & & & & $P=0.313$ & $P=0.108$ & $P=0.352$ & $P=0.028$ & $P=0.020$ & $P=0.009$ & $P=0.002$ & $P=0.001$ & $P=0.001$ & \\
\hline & \multirow{6}{*}{$\begin{array}{l}\text { w/o } \\
\text { gel }\end{array}$} & & & 4.83 & 5.05 & 4.77 & 4.27 & 4.22 & 4.05 & 3.77 & 3.33 & 1.0 & \\
\hline & & $24^{\circ} \mathrm{C}$ & 5.22 & (-7.5\%) & $(-3.3 \%)$ & $(-8.6 \%)$ & (-18.2\%) & $(-19.2 \%)$ & $(-22.4 \%)$ & (-27.8\%) & $(-36.2 \%)$ & $(-80.8 \%)$ & \\
\hline & & & & $P=0.001$ & $P=0.002$ & $P=0.001$ & $P=0.001$ & $P=0.001$ & $P=0.001$ & $P=0.001$ & $P=0.001$ & $P=0.001$ & \\
\hline & & & & 4.88 & 4.77 & 4.61 & 4.38 & 4.27 & 4.22 & 4.00 & 3.84 & 3.55 & \\
\hline & & $4^{\circ} \mathrm{C}$ & 5.22 & $(-6.5 \%)$ & (-8.6\%) & (-11.7\%) & (-16.1\%) & $(-18.2 \%)$ & (-19.2\%) & $(-23.4 \%)$ & $(-26.4 \%)$ & $(-32 \%)$ & \\
\hline & & & & $P=0.001$ & $P=0.001$ & $P=0.001$ & $P=0.001$ & $P=0.001$ & $P=0.001$ & $P=0.001$ & $P=0.001$ & $P=0.001$ & \\
\hline
\end{tabular}




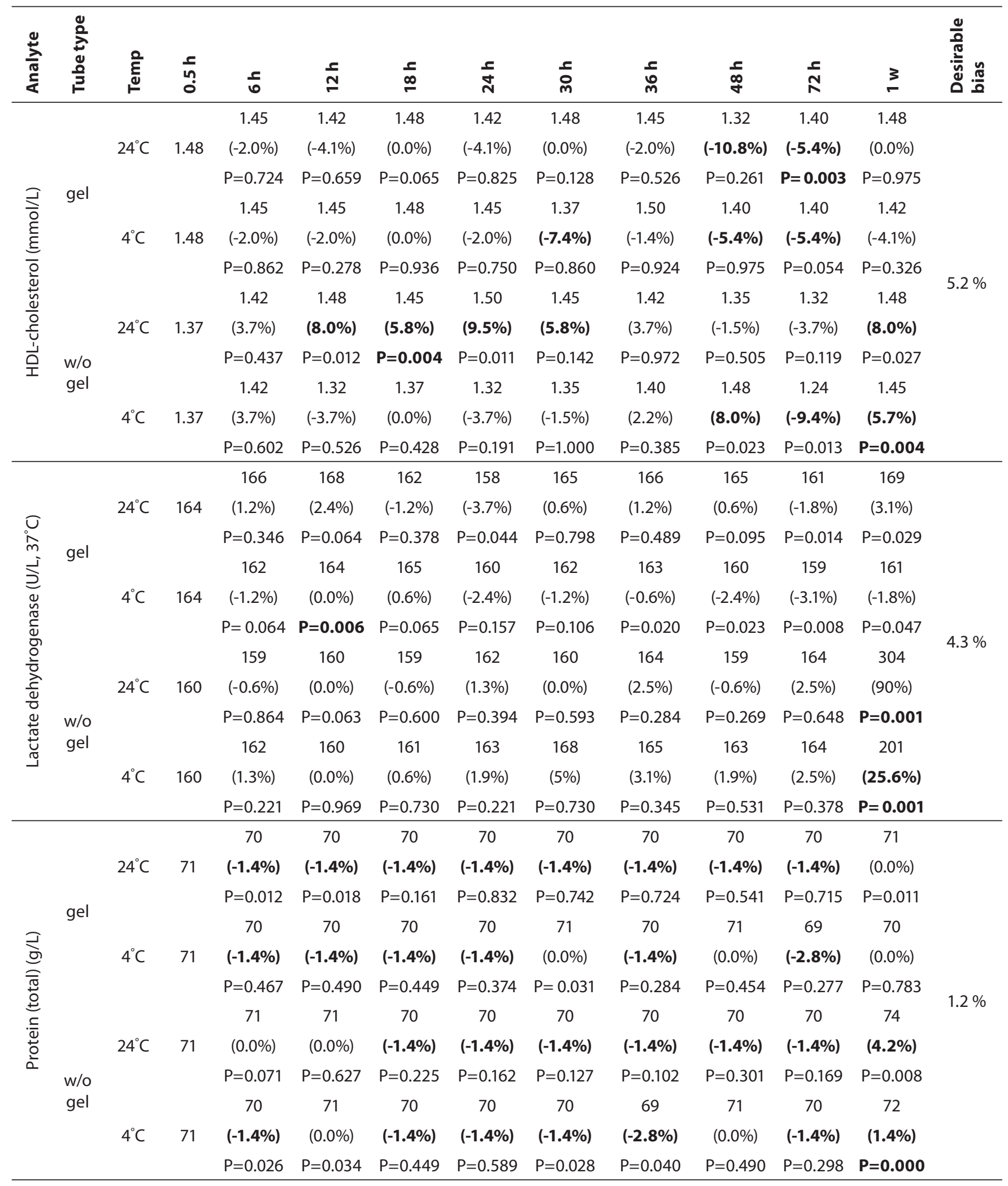




\begin{tabular}{|c|c|c|c|c|c|c|c|c|c|c|c|c|c|}
\hline 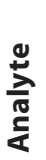 & 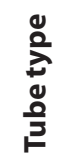 & 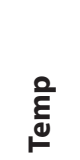 & $\begin{array}{l}\frac{c}{n} \\
0 \\
0\end{array}$ & $\frac{1}{0}$ & $\frac{\mathbf{c}}{\mathbf{N}}$ & $\underset{\infty}{\stackrel{\infty}{\infty}}$ & $\frac{\check{d}}{\mathbf{N}}$ & 일 & $\frac{c}{b}$ & $\underset{\stackrel{\infty}{+}}{\stackrel{c}{+}}$ & $\stackrel{\mathbf{N}}{\mathbf{N}}$ & $\frac{3}{6}$ & $\begin{array}{l}\frac{0}{0} \\
\frac{0}{\frac{2}{n}} \\
\frac{\pi}{0} \\
\frac{0}{0}\end{array}$ \\
\hline \multirow{12}{*}{ 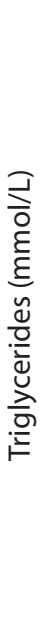 } & \multirow{6}{*}{ gel } & & & 0.84 & 0.86 & 0.87 & 0.83 & 0.86 & 0.87 & 0.84 & 0.86 & 0.88 & \multirow{12}{*}{$10.7 \%$} \\
\hline & & $24^{\circ} \mathrm{C}$ & 0.83 & $(1.2 \%)$ & (3.6\%) & (4.8\%) & $(0.0 \%)$ & (2.7\%) & $(4.8 \%)$ & $(1.2 \%)$ & (3.6\%) & $(6.0 \%)$ & \\
\hline & & & & $P=0.059$ & $P=0.145$ & $P=0.004$ & $P=0.009$ & $P=0.062$ & $P=0.001$ & $P=0.001$ & $P=0.001$ & $P=0.001$ & \\
\hline & & & & 0.89 & 0.88 & 0.91 & 0.86 & 0.86 & 0.83 & 0.86 & 0.87 & 0.89 & \\
\hline & & $4^{\circ} \mathrm{C}$ & 0.83 & $(7.2 \%)$ & $(6.0 \%)$ & $(9.6 \%)$ & (3.6\%) & (3.6\%) & $(0.0 \%)$ & (3.6\%) & $(4.8 \%)$ & (7.2\%) & \\
\hline & & & & $P=0.001$ & $P=0.193$ & $P=0.001$ & $P=0.001$ & $P=0.001$ & $P=0.001$ & $P=0.001$ & $P=0.001$ & $P=0.001$ & \\
\hline & \multirow{6}{*}{$\begin{array}{l}\text { w/o } \\
\text { gel }\end{array}$} & & & 0.87 & 0.87 & 0.87 & 0.88 & 0.88 & 0.90 & 0.89 & 0.90 & 0.94 & \\
\hline & & $24^{\circ} \mathrm{C}$ & 0.86 & $(1.2 \%)$ & (1.2\%) & $(1.2 \%)$ & $(2.3 \%)$ & (2.3\%) & $(4.7 \%)$ & (3.5\%) & $(4.7 \%)$ & $(9.3 \%)$ & \\
\hline & & & & $P=0.002$ & $P=0.002$ & $P=0.001$ & $P=0.001$ & $P=0.010$ & $P=0.001$ & $P=0.001$ & $P=0.001$ & $P=0.001$ & \\
\hline & & & & 0.86 & 0.87 & 0.88 & 0.86 & 0.87 & 0.89 & 0.89 & 0.88 & 0.90 & \\
\hline & & $4^{\circ} \mathrm{C}$ & 0.83 & (3.6\%) & (4.8\%) & (6.0\%) & (3.6\%) & (4.8\%) & (7.2\%) & (7.2\%) & (6.0\%) & (8.4\%) & \\
\hline & & & & $P=0.015$ & $P=0.002$ & $P=0.001$ & $P=0.001$ & $P=0.001$ & $P=0.001$ & $P=0.001$ & $P=0.001$ & $P=0.001$ & \\
\hline \multirow{12}{*}{ 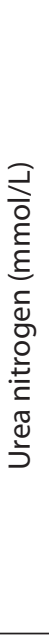 } & \multirow{6}{*}{ gel } & & & 4.29 & 4.29 & 3.93 & 4.29 & 4.29 & 4.29 & 3.93 & 4.64 & 4.64 & \multirow{12}{*}{$5.5 \%$} \\
\hline & & $24^{\circ} \mathrm{C}$ & 4.64 & $(-6.7 \%)$ & $(-6.7 \%)$ & $(-15.3 \%)$ & $(-6.7 \%)$ & (-6.7\%) & $(-6.7 \%)$ & $(-15.3 \%)$ & $(0.0 \%)$ & $(0.0 \%)$ & \\
\hline & & & & $P=0.206$ & $P=0.046$ & $P=0.084$ & $P=0.161$ & $P=0.109$ & $P=0.331$ & $P=0.218$ & $P=0.062$ & $P=0.083$ & \\
\hline & & & & 4.64 & 4.64 & 4.64 & 4.64 & 4.64 & 4.29 & 4.64 & 5.00 & 4.64 & \\
\hline & & $4^{\circ} \mathrm{C}$ & 4.64 & (0.0\%) & (0.0\%) & (0.0\%) & (0.0\%) & (0.0\%) & (-7.8\%) & $(0.0 \%)$ & (7.8\%) & (0.0\%) & \\
\hline & & & & $P=0.776$ & $P=0.053$ & $P=0.102$ & $P=0.339$ & $P=0.248$ & $P=1.000$ & $P=0.564$ & $P=0.001$ & $P=0.177$ & \\
\hline & \multirow{6}{*}{$\begin{array}{l}\text { w/o } \\
\text { gel }\end{array}$} & & & 4.29 & 4.29 & 4.64 & 4.64 & 4.64 & 4.29 & 4.64 & 5.00 & 5.36 & \\
\hline & & $24^{\circ} \mathrm{C}$ & 4.29 & (0.0\%) & (0.0\%) & $(8.2 \%)$ & (8.2\%) & (8.2\%) & (0.0\%) & (8.2\%) & (16.6\%) & $(25 \%)$ & \\
\hline & & & & $P=1.000$ & $P=0.942$ & $P=0.130$ & $P=0.206$ & $P=0.796$ & $P=0.1000$ & $P=0.083$ & $P=0.002$ & $P=0.001$ & \\
\hline & & & & 4.64 & 4.29 & 4.64 & 4.64 & 4.64 & 4.29 & 4.64 & 5.00 & 5.00 & \\
\hline & & $4^{\circ} \mathrm{C}$ & 4.29 & (8.2\%) & (0.0\%) & (8.2\%) & (8.2\%) & (8.2\%) & (0.0\%) & $(8.2 \%)$ & (16.6\%) & (16.6\%) & \\
\hline & & & & $P=0.564$ & $P=0.958$ & $P=0.564$ & $P=0.298$ & $P=0.963$ & $P=0.856$ & $\mathrm{P}=0.080$ & $P=0.001$ & $P=0.006$ & \\
\hline \multirow{12}{*}{ 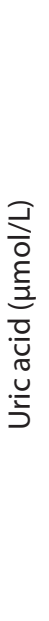 } & \multirow{6}{*}{ gel } & & & 238 & 250 & 244 & 244 & 250 & 256 & 262 & 274 & 274 & \multirow{12}{*}{$4.9 \%$} \\
\hline & & $24^{\circ} \mathrm{C}$ & 232 & $(2.6 \%)$ & (7.8\%) & (5.2\%) & (5.2\%) & (7.8\%) & (10.3\%) & (12.9\%) & (18.1\%) & (18.1\%) & \\
\hline & & & & $P=0.058$ & $P=0.001$ & $P=0.001$ & $P=0.001$ & $P=0.001$ & $P=0.001$ & $P=0.001$ & $P=0.001$ & $P=0.001$ & \\
\hline & & & & 226 & 232 & 238 & 238 & 232 & 244 & 244 & 250 & 244 & \\
\hline & & $4^{\circ} \mathrm{C}$ & 232 & 0.003 & (0.0\%) & (2.6\%) & (2.6\%) & (0.0\%) & (5.2\%) & (5.2\%) & (7.8\%) & (5.2\%) & \\
\hline & & & & $P=0.033$ & $P=0.446$ & $P=0.296$ & $P=0.444$ & $P=0.145$ & $P=0.034$ & $P=0.015$ & $P=0.003$ & $P=0.001$ & \\
\hline & \multirow{6}{*}{$\begin{array}{l}\text { w/o } \\
\text { gel }\end{array}$} & & & 238 & 244 & 256 & 244 & 250 & 262 & 256 & 268 & 286 & \\
\hline & & $24^{\circ} \mathrm{C}$ & 232 & (2.6\%) & (5.2\%) & (10.3\%) & (5.2\%) & (7.8\%) & (12.9\%) & (10.3\%) & (15.5\%) & (23.3\%) & \\
\hline & & & & $P=0.003$ & $P=0.001$ & $P=0.001$ & $P=0.002$ & $P=0.004$ & $P=0.001$ & $P=0.001$ & $P=0.001$ & $P=0.001$ & \\
\hline & & & & 232 & 232 & 238 & 220 & 226 & 220 & 232 & 238 & 256 & \\
\hline & & $4^{\circ} \mathrm{C}$ & 232 & (0.0\%) & (0.0\%) & (2.6\%) & $(-5.2 \%)$ & $(-2.6 \%)$ & $(-5.2 \%)$ & (0.0\%) & $(2.6 \%)$ & (10.3\%) & \\
\hline & & & & $P=0.157$ & $P=0.053$ & $P=0.014$ & $P=0.001$ & $P=0.010$ & $P=0.763$ & $P=0.672$ & $P=0.002$ & $P=0.001$ & \\
\hline
\end{tabular}

${ }^{*}$ without gel.

$\mathrm{P}<0.006$ values were written in bold font. Percentages in parenthesis are bias values of specific time points compared to initial value ( $0.5 \mathrm{~h})(\%$ change from $T_{0}(0.5-h)$ ).

Bold font indicates samples for which the differences exceeded the maximum desirable bias. 
based on clinically meaningful variations, as reflected by deviation from the current analytical quality specifications for desirable bias (10). Albumin and total protein concentrations did not show any difference significantly up to $72-\mathrm{h}$ in all groups.

Time-course plots for selected analytes that illustrate various stability situations are presented in Figure 1. Glucose, uric acid, BUN, and HDL were found as the least stable tests. Glucose concentrations decreased markedly beginning from the first
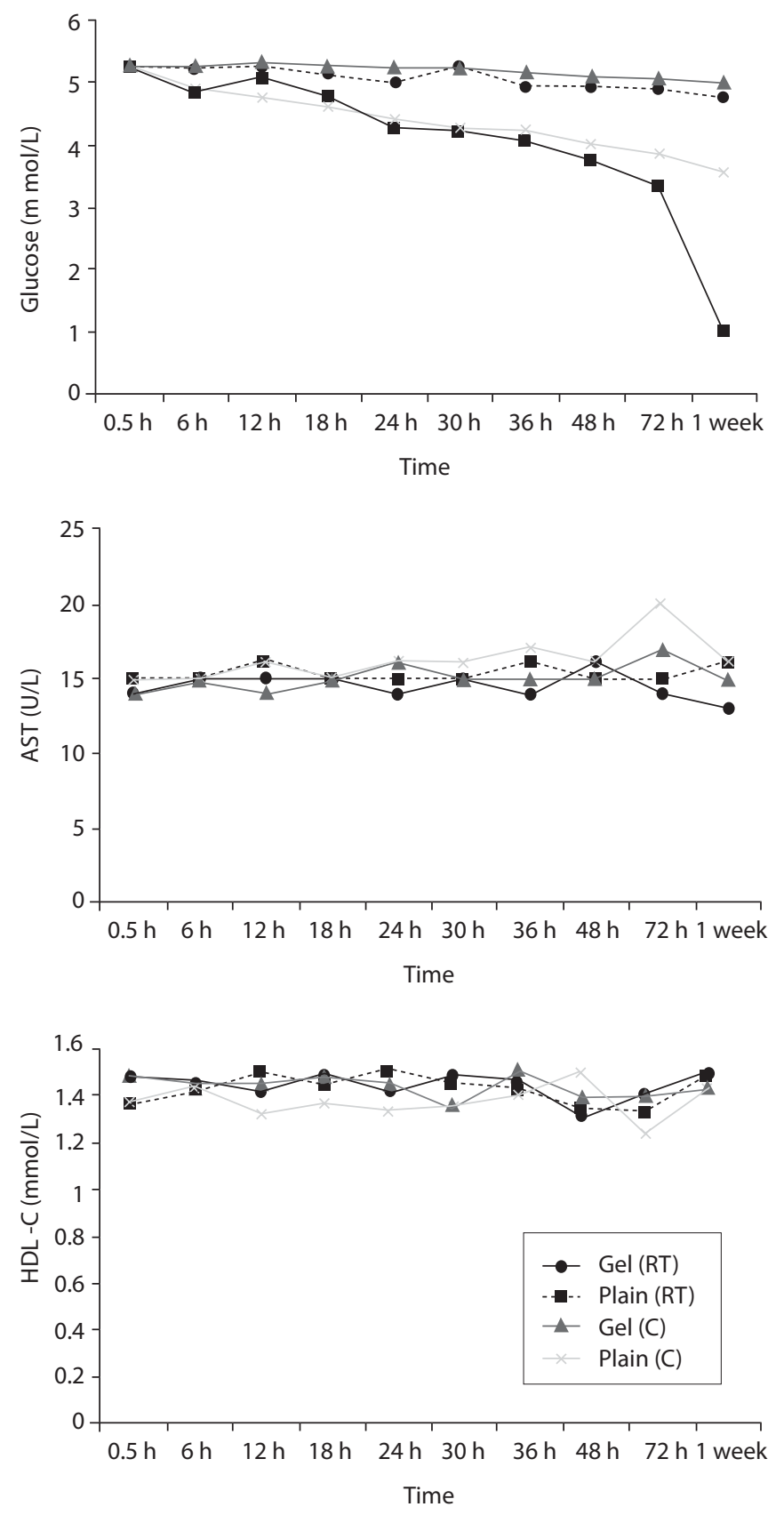

hours of storage especially in plain serum (by more than $18 \%$ just in one day) at RT. HDL was the least stable when stored at room temperature in plain serum. BUN and uric acid were stable only in chilled serum with gel barrier as long as $30-\mathrm{h}$. AST increased only one or two units up to 48-h of storage in all four groups. The percentage changes in total bilirubin were under the clinically meaningful variations when stored in serum gel tubes either at RT or chilled up to one week (Table 3). There were no failures in barrier formation in the gel tubes during the study.
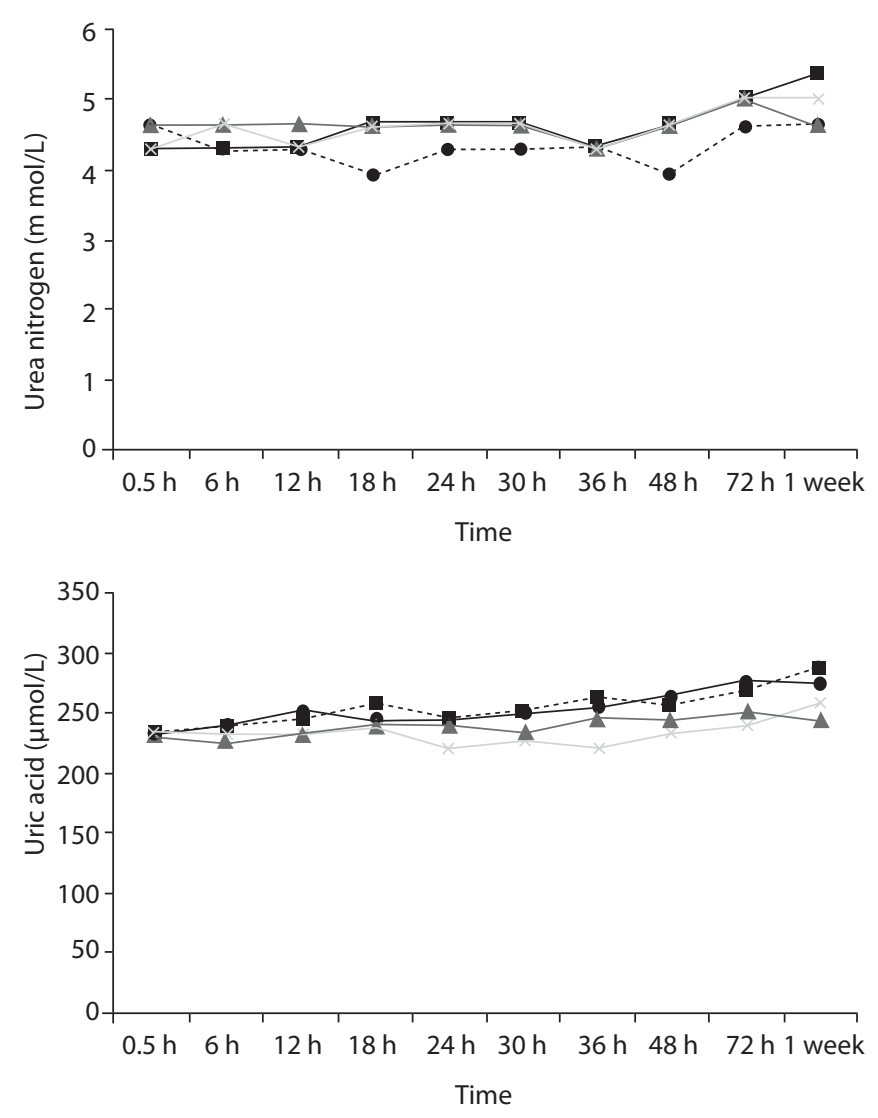

Figure 1. Time and temperature dependency of glucose, uric acid, BUN, HDL-C concentrations and AST activity on storage of serum in tube with (gel) and without gel (plain). RT - room temperature; C - Chilled. 


\section{Discussion}

Contrary to possible expectations, the wide range of biochemical analytes that are stored in separator tubes for several days was more stable than had already been believed. The current study demonstrates that concentrations of albumin, total protein, creatinine, cholesterol, triglycerides and activities of GGT, ALP, ALT, CK, LD did not differ either in serum separator gel tube or in plain tube and can be measured reliably in samples kept at RT or at $4{ }^{\circ} \mathrm{C}$ for at least three days after centrifugation. Glucose concentrations decreased with increasing serum-clot contact time in plain tube and the rate of decrease was sensitive to temperature like previous studies $(11,12)$. This decrease was attributed to glycolytic action of erythrocytes and leukocytes $(13,14)$ cited by Tietz $(15)$. The rate of decrease may be minimized when refrigerated at $4{ }^{\circ} \mathrm{C}$. BUN demonstrated a better performance with a gel barrier like a previous study (16) where the increase in BUN was attributed to hemoconcentration in serum plain at both temperatures.

The stability of total bilirubin in gel tubes over time demonstrated the efficiency of gel barrier regardless of the temperature in the current study. Similar to the study from Heins et al. (17), the concentrations of total bilirubin showed a decreasing trend stored in plain serum at RT.

In general, uric acid instability was prominent at RT like previous studies $(17,18)$, and gel tube had no advantages over plain tube.

During the experimental study, creatinine showed decreases only in concentrations from 18 to 24-h with gel tube at RT, and 48 to $72-\mathrm{h}$ with chilled plain tube exceeding the desirable bias; however, these $8.8 \mu \mathrm{mol} / \mathrm{L}$ decreases may not be considered clinically significant. Similar results were obtained by Heins et al. (17). Besides, in contrast to the previous study (16), we demonstrated that gel tube has no advantage over plain tube for creatinine assay. In the current study, small changes in concentrations were observed for albumin and total protein which were outside the maximum desirable bias (10). However, the analytical and biological variations of those analytes are very small, for that reason we considered the changes in concentrations as clinically insignificant.
Cholesterol and triglycerides were stable up to one week in the current study. Similar results were obtained by Boyanton and Blick (18).

For $\mathrm{HDL}$, gel tubes at RT and, plain tubes at $4{ }^{\circ} \mathrm{C}$ displayed similar results and were stayed stable up to 36-h. However, in plain tube at RT, HDL concentrations increased by $8.8 \%$ at $12-\mathrm{h}$ to a level that exceeded the clinically significant value. O'Keane and Cunningham (16) reported non significant changes up to 48-h in serum gel and in serum plain at RT or at $4{ }^{\circ} \mathrm{C}$. Heins et al. (17) reported increases in HDL concentrations in plain serum at RT, but found as stable at $9^{\circ} \mathrm{C}$.

Since there are discrepancies between the results of other studies and the present study, it might be explained by differences in analytic methods, by different storage temperatures and assay intervals as most of them assayed in the same run, however in the current study all were assayed at different time points like Heins et al. (17).

The enzymes GGT, ALP, CK and ALT were found as stable up to 72 -h of storage in all groups. Heins et al. (17) found the same stability in plain serum up to one week at RT except for CK.

LD activity was stable as long as one week in serum gel tube at both temperatures.

The impressive peaks in the activity of LD at one week in plain sera may be due to interference of hemolysis (19-21) caused by changes in cell permeability, and to the fragility of erythocyte membrane during the prolonged storage. Sodi et al. (22) examined the effect of sample transportation on possible hemolysis, and concluded the advantages of gel barrier tubes over plain tubes. Heins et al. (17) also emphasized the instability of LD in whole blood.

In this study, AST activity changed only a few units despite the marked percentage biases and may not be considered as clinically significant as long as 48-h of storage in all four groups. Heins et al. (17) also noted the stability of AST stayed until one week at RT.

For total bilirubin, glucose, BUN, uric acid and LD, gel tubes showed enhanced stability compared to plain tubes and the stability can be maximized by refrigeration at $4^{\circ} \mathrm{C}$. Practically, gel tubes can be preferred for prolonged storage, for protection 
against possible contamination while aliquoting and for possible cell leakage.

There are two limitations that need to be acknowledged regarding the present study. The first limitation concerns the visible detection of the possible hemolysis. We have not studied the free hemoglobin concentrations in the sera stored during the process. So, we have only attributed the biases of LD to the possible hemolysis interference. Besides, the evaluation of the storage conditions were done on a defined analyzer with its corresponding reagents with the samples stored in defined tube types. Therefore, the results of the current study might not be universally reproducible with other testing systems for the evaluation of the stabilities of samples stored for a time period before analysis.

\section{References}

1. Lippi G, Chance JJ, Church S, Dazzi P, Fontana R, Giavarina $D$, et al. Preanalytical quality improvement: from dream to reality. Clin Chem Lab Med 2011;49:1113-26.

2. Lippi G, Guidi GC, Mattiuzzi C, Plebani M. Preanalytical variability: the dark side of the moon in laboratory testing. Clin Chem Lab Med 2006;44:358-65.

3. Lippi G, Simundic AM. Total quality in laboratory diagnostics. It's time to think outside the box. Biochem Med 2010;20:5-8.

4. Lippi G, Plebani M, Simundic AM. Quality in laboratory diagnostics: from theory to practice. Biochem Med 2010;20:126-30.

5. Lippi G, Simundic AM, Mattiuzzi C. Overview on patient safety in healthcare and laboratory diagnostics. Biochem Med 2010;20:131-43.

6. Bowen RA, Hortin G, Csako G, Otañez OH, Remaley AT. Impact of blood collection devices on clinical chemistry assays. Clin Biochem 2010;43:4-25.

7. Wilde C. Subject preparation, sample collection, and handling. In: Wild D, editor. The immunoassay handbook. New York: Elsevier; 2005. p. 243-55.

8. Bowen RA, Chan Y, Ruddel ME, Hortin GL, Csako G, Demosky SJ, et al. Immunoassay interference by a commonly used blood collection tube additive, the organosilicone surfactant silwet L-720. Clin Chem 2005;51:1874-82.

9. Dimeski G. Interference testing. Clin Biochem Rev 2008;29:43-8.

10. Westgard QC. Desirable specifications for total error, imprecision, and bias, derived from intra- and inter - individual biological variation. Available at: http://www.westgard. com/biodatabase1.htm\#11. Accessed January 2012.
In conclusion, in the current study, we have shown that the concentrations of many analytes in sera in separator tube with gel or without gel did not show any significant changes even at RT as long as three days. Therefore, those mentioned analytes may be measured reliably in mailed blood samples. However it is important to note that, in hotter climates, (higher than the defined room temperature in this study), if the samples needed to be stored for long periods until analysis, gel tubes and storage with cold packs may be a better option.

\section{Potential conflict of interest}

None declared.

11. Giampietro O, Navalesi R, Buzzigoli G, Boni C, Benzi L. Decrease in plasma glucose concentration during storage at -20 ${ }^{\circ} \mathrm{C}$. Clin Chem 1980;26:1710-2.

12. Dirar A, Dalael AA, Abdelsalam AK. Effect of storage time and temperature on some serum analytes. International Journal of Pathology 2010;8:68-71.

13. Chan AYW, Swaminathan R, Cockram CS. Effectiveness of sodium fluoride as a preservative of glucose in blood. Clin Chem 1989;35:315-7.

14. Weisman $M$, Klein B. Evaluation of glucose determination in untreated serum samples. Clin Chem 1986;32:1544-8.

15. Sachs DB. Carbohydrates. In: Burtis CA, As hwood ER, eds. Tietz Textbook of Clinical Chemistry and molecular diagnostics. $5^{\text {th }}$ ed. Philadelphia: WB Saunders Company; 2012.p.718-21.

16. O'Keane MP, Cunningham SK. Evaluation of three different specimen types (serum, plasma lithium heparin and serum gel separator) for analysis of certain analytes: clinical significance of differences in results and efficiency in use. Clin Chem Lab Med 2006;44:662-8.

17. Heins $M$, Heil W, Withold W. Storage of serum or whole blood samples? Effects of time and temperature on 22 serum analytes. Eur J Clin Chem Clin Biochem 1995;33:231-8.

18. Boyanton BL, Blick K. Stability studies of twenty-four analytes in human plasma and serum. Clin Chem 2002;48:2242-7.

19. Lippi G, Blanckaert N, Bonini P, Green S, Kitchen S, Palicka V, et al. Haemolysis: an overview of the leading cause of unsuitable specimens in clinical laboratories. Clin Chem Lab Med 2008;46:764-72. 
20. Simundic AM, Topic E, Nikolac N, Lippi G. Hemolysis detection and management of hemolyzed specimens. Biochem Med 2010;20:154-9.

21. Koseoglu M, Hur A, Atay A, Cuhadar S. Effects of hemolysis interference on routine biochemistry parameters. Biochem Med 2011;21:79-85.
22. Sodi R, Darn SM, Stott A. Pneumatic tube system induced haemolysis: assessing sample type susceptibility to haemolysis. Ann Clin Biochem 2004;41:237-40.

23. Koseoglu M, Isleten F, Dursun S, Cuhadar S. Determination of reference intervals of healthy adults aged between 20-50 years in Izmir. Turk J Biochem 2010;35:215-224.

\section{Ispitivanje stabilnosti biokemijskih analita u serumskim epruvetama s ili bez gela za odvajanje u različitim uvjetima pohranjivanja}

\section{Sažetak}

Uvod: Sakupljeni i transportirani uzorci krvi često su prije same analize izloženi različitim izvananalitičkim čimbenicima. Cilj ovog ispitivanja bio je utvrditi stabilnost analita u epruvetama za odvajanje seruma s gelom i u običnim epruvetama koje su nakon centrifugiranja bile izložene širokom rasponu temperatura i vremena pohranjivanja.

Materijali i metode: $U$ istraživanju je sudjelovalo 15 dobrovoljaca od kojih je sakupljena venska krv u četiri epruvete, dvije s gelom za razdvajanje seruma, a dvije bez gela. Nakon analize uzoraka te određivanja početnih vrijednosti 30 minuta nakon uzimanja krvi, svi su uzorci pohranjeni na $4{ }^{\circ} \mathrm{C}$ ili $24^{\circ} \mathrm{C}$ tijekom 6, 12, 18, 24, 30, 36, 48, 72 sata i jedan tjedan. U svakom je uzorku određena koncentracija 16 biokemijskih parametara. Varijacije ispod željene sustavne pogreške smatrane su klinički neznačajnima.

Rezultati: Trećeg je dana većina analita ostala stabilnima uključujući albumin, protein, kreatinin, kolesterol, trigliceride, gama-glutamil-transferazu (GGT), alkalnu fosfatazu (ALP), kreatin-kinazu (CK), laktat-dehidrogenazu (LD) bez obzira na vrstu epruvete. Stabilnost glukoze, pohranjene u epruveti bez gela (u čistom serumu) značajno je smanjenja $(P=0,001)$ već nakon prvih sati pohranjivanja. Najveća je stabilnost primjećena kod analita pohranjenih na $4{ }^{\circ} \mathrm{C}$ u epruvetama s gelom i to kod glukoze, ukupnog bilirubina, ureje (BUN) i mokraćne kiseline. Aktivnost aspartat-aminotransferaze (AST) značajno je porasla $(P=0,002)$ kod uzoraka pohranjenih do 48 sati, međutim sustavna pogreška nije bila klinički značajna. Koncentracija HDL kolesterola bila je stabilna u epruvetama s gelom na $24^{\circ} \mathrm{C}$ te u običnim epruvetama na $4^{\circ} \mathrm{C}$ do 36 sati.

Zaključak: Epruvete za odvajanje seruma s ili bez gela mogu se primjenjivati naizmjenično za 11 analita ohlađenih na $4^{\circ} \mathrm{C}$ ili na temperaturi od $24^{\circ} \mathrm{C}$, dok kod određivanja koncentracije glukoze, HDL kolesterola, mokraćne kiseline, uree i aktivnosti AST trebaju postojati neka ograničenja.

Ključne riječi: uzorkovanje; stabilnost enzima; pohranjivanje; temperatura 